Un nouveau cours de formation continue en gestion axé sur la pratique, développé par la SSMI et la FHNW à l'intention des médecins et des cadres médicaux

\title{
CAS Management of Medical Units
}

Les médecins désireux de faire carrière en hôpital ou en cabinet médical doivent assumer toujours plus de tâches de gestion. Or le cursus médical ne les y prépare pas suffisamment. Afin de développer les compétences des médecins dans cette matière, la Société Suisse de Médecine Interne Générale (SSMI) s'est associée à la Haute école spécialisée du Nord-Ouest de la Suisse FHNW pour organiser à partir d'avril 2013 un cours de formation en gestion dirigé vers la pratique: le CAS Management of Medical Units.

Jean-Michel Gaspoz ${ }^{a}$,

Lukas Zemp ${ }^{b}$,

Volker Bernhard Schultec

a Prof., président du directoire de la SSM

b Secrétaire général/directeur du bureau administratif de la SSMI

c Prof., Head Competence Center Health Management, Haute école de gestion FHNW

\footnotetext{
Fachhochschule Nordwestschweiz
}

Correspondance: Société Suisse de Médecine Interne Générale (SSMI) Solothurnerstrasse 68 Case postale 422 CH-4008 Bâle Tél. 0612259330 Fax 0612259331 sgim[at]sgim.ch
Pour être à même de fonctionner comme cadre dans un hôpital ou comme décideur dans un cabinet de groupe, ou encore pour réussir en tant qu'indépendant dans un cabinet médical, le médecin doit de plus en plus souvent dépasser les domaines généraux et spécialisés de la médecine et posséder des compétences de gestionnaire et de directeur. Mais actuellement, il n'a généralement bénéficié d'aucune formation dans ce domaine. Pour combler cette lacune, la Société Suisse de Médecine Interne Générale (SSMI) a mis sur pied un nouveau certificat de formation continue en gestion de la santé, le CAS (Certificate of Advanced Studies) Management of Medical Units, en collaboration avec la Haute école spécialisée du NordOuest de la Suisse $\left(\mathrm{FHNW}^{*}\right)$.

\section{Un cours que le médecin peut intégrer dans son programme de travail et qui s'avère très utile}

Le cours menant au certificat CAS Management of Medical Units a été élaboré conjointement par la SSMI et la Haute école de gestion FHNW. Elaboré sur mesure pour les médecins et les cadres médicaux, il comprend 14 jours de formation répartis entre avril et août 2013. Les candidats doivent s'inscrire avant la fin du mois de mars 2013. Le cours sera dispensé pour la première fois en Suisse en 2013 sous forme de projet pilote conçu pour la Suisse alémanique. Il est construit sur la base d'une formation largement reconnue à l'échelle internationale, l'Executive Master in Business Administration EMBA de la Haute école de gestion FHNW, et il tient compte des besoins spécifiques des médecins exerçant en ambulatoire ou à l'hôpital. Le CAS Management of Medical Units permet d'acquérir des connaissances générales en gestion des entreprises et d'approfondir certains sujets spécifiques à la gestion de la santé.

\section{Des points qui comptent: \\ les crédits ECTS et ISFM}

Les détenteurs du nouveau certificat ont droit à 15 points ECTS. Le système ECTS (European Credit Transfer and Accumulation System) vise à promouvoir la transparence entre les différents systèmes de formation européens. De plus, la formation continue élargie donne droit à 25 crédits ISFM par année, soit au maximum 75 crédits ISFM dans le cadre d'une période de formation continue de trois ans.

D'autres informations figurent aux adresses www. fhnw.ch/wirtschaft/weiterbildung/cas ou www.ssmi. $\mathrm{ch} / \mathrm{fr} / \mathrm{manifestations-de-formation-continue.}$

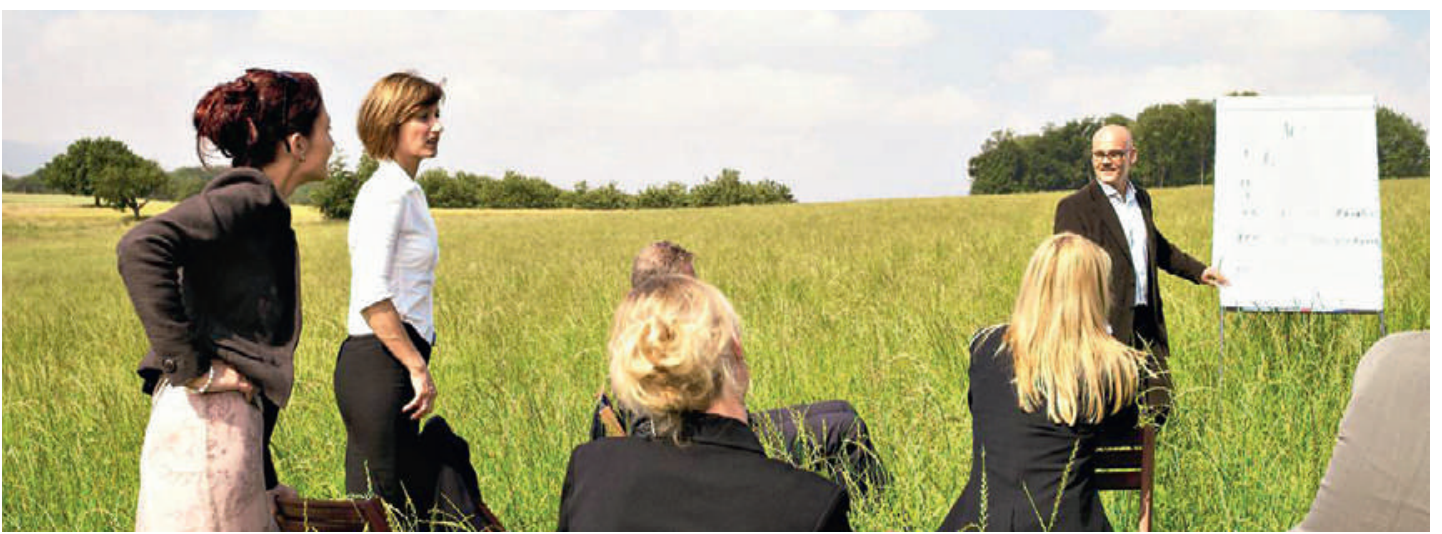

